Pak. j. sci. ind. res. Ser. A: phys. sci. 2020 63A(3) 231-238

\title{
Review
}

\section{A Brief Review of Synthesis, Properties and Applications of Graphene}

\author{
Tahir Iqbal, Ahmad Raza, Almas Bashir* and Aqsa Tehseen \\ Department of Physics, University of Gujrat, Hafiz Hayat Campus, Gujrat, 50700, Pakistan
}

(received December 7, 2018; revised September 9, 2019; accepted September 17, 2019)

\begin{abstract}
At time when the limitation of silicon capabilities is being touched the finding of graphene and its exclusive properties of nano-scale are of utmost importance. The possible substitutes of following generation for quicker and minor electronics of present $21^{\text {st }}$ century. Several methods and techniques are being experimented to produce graphene with enhanced properties to be used as a substitute for existing materials. Raman spectrophotometry, X-ray diffraction, field emission-scanning electron microscopy (FE-SEM) and tunneling electron microscopy are the characterizing techniques for the prepared graphene. Graphene is the core compound of interest for scientists for this decade and previous as well, because of its exceptional impact on many life disciplines. That is why the increasing trend in recent years for the graphene and graphene oxides is observed.
\end{abstract}

Keywords: graphene nano sheets, precursors, dry exfoliation, chemical vapour deposition (CVD)

\section{Introduction}

Ijma prepared and introduced carbon nanotubes in 1991. Later in 2009, Andre Geim manufactured graphene as a single two-dimensional plane of graphite but it was a theoretical material as it could not be produced in excessive amount. (Geim, 2009) Graphene, as marvel material, consist of monolayer hexagonal $\mathrm{sp}^{2}$ hybridized carbon atoms. It is a single known thinnest sheet of graphite. It has the perfect 2D arrangement. (Hansora et al., 2015).

Graphene has developed as a new super material because of its exclusive physical properties. These novel twodimensional nanostructures has engrossed marvelous attention equally from basic and applied research aspects in fields of physics, chemistry and materials science as well. (Sutter et al., 2008) Nowadays, graphene is of greatest attraction nanomaterial not only due to its thinnest structure among known materials in the universe or of as it is toughest forever measured material as well but also because of its exceptional properties. (Rao et al., 2009). Graphene is an ultimate personification of surface as it has two surfaces having no bulk among them, while the surface's physics is now at the focus of attention, its chemistry has not been fundamentally explored. So far about its chemistry we have only

*Author for correspondence;

E-mail: tiqbal02@qub.ac.uk; almasbashir001@gmail.com learned that, just like the surface of graphite, the nanocomposites of graphene can adsorb and desorb many atoms and molecules (Booth et al., 2008).

The two-dimensional honeycomb like structure for graphene sheet is given in Fig. 1. In the graphene sheet, the atoms are arranged in a hexagonal fashion with twodimensional structure.

The monolayer atom having dense flat-bed arrangement has transformed nanotechnology platform from the time of its discovery. Numerous tries have been finished to

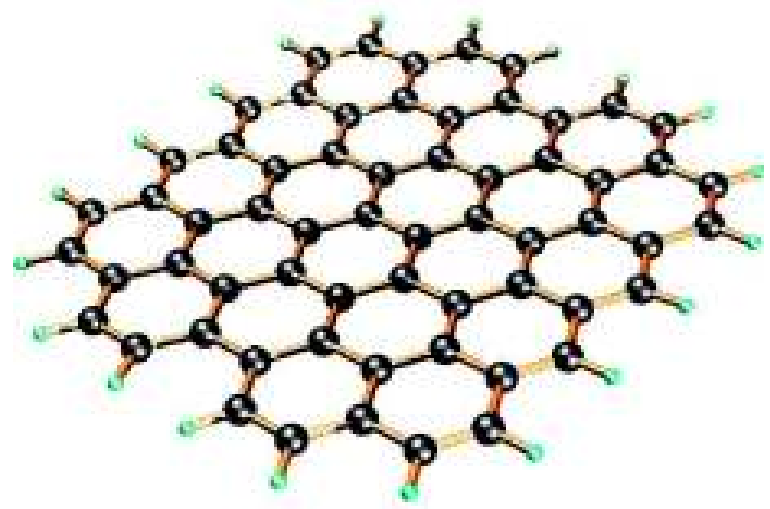

Fig. 1. Execution of 2 D sheet of Graphene, displaying hexagonal, honeycomb-like arrangement (Somani and Pawer, 2016). 
produce graphene on larger scale to accomplish the requirements of numerous industries, mostly in fused industry, where the usage of graphene has intensely changed international marketplace for the manufacturing of state-of-the- art complex constituents. The specific and distinct characteristics of graphene named it as "magic bullet" for the compound world. (Gautam et al., 2012). Alongwith number of advantages such as extraordinary surface-to-volume ratio for assembly of graphene oxide nanosheets, numerous sheets are extensively prepared and have shown excessive potential in various membrane parting fields such as gas separation and desalination (Hung et al., 2014). Carbon nanomaterials related to graphene are of different types as doped graphene, graphene derivative nanoribbons, graphene oxide and porous graphene. To reduce the size for data storing devices or to increase the capability of these devices, while sustaining the size of the hardware, is less studied in the World graphene. Investigation on storage capabilities of graphene oxides has shown that the electrodes adapted by oxides of polymers and graphene show the write-read-erase and read-rewrite succession for nonvolatile memorial device. (Randviir et al., 2014) It may be bounded in zero dimensional fullerenes, moved in one dimensional nanotubes which form stacks in 3D graphite. In this way, graphene is known as a base for all carbon-based nanomaterials which are graphitic in nature and has a splendid potential in practical fields of science (Ma $e t$ al., 2017).

This review is mainly focusing on scope and application of graphene in multiple fields of life. This will provide detail information, especially for research beginners in the field of development and improvements of graphene and its capabilities. A vast range of its uses and scope in daily life is of great interest. The important method of synthesis along with detail constituents, precursor, catalysts and techniques used in its manufacturing and also mentioned here. It will also provide a guideline to new workers for focusing on its improved synthetic form and the characterizing techniques which can be applied to understand its structural informations.

Earlier trend of growth in graphene study. Right from its discovery, graphene has transformed and fully redefining the current day technology due to its amazing properties. The research about its potential and discovery has exponentially developed by several universities, R\&D institutions' and numerous private and administrative groups round the World. Study on graphene is found in all fields today. An overview of several available research works, which illustrates the incentives in graphene research around the globe is presented by graphs since 2012. The worldwide number of publications with the use of web science tool, a vibrant representation of the publication trend for graphene is shown in graphs below in Fig. 2 (Kim et al., 2010).

Country wise number of publications with the use of web science tool, a vibrant representation of the publication trend for graphene is shown in graphical representation in Fig. 3 below (Kim et al., 2010).

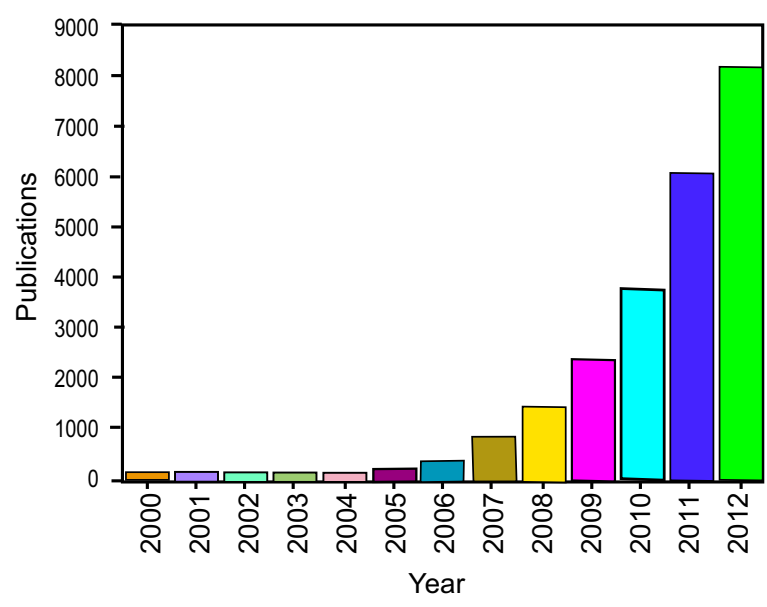

Fig. 2. Publication development in graphene since 2000-2012 (Dhand et al., 2013).

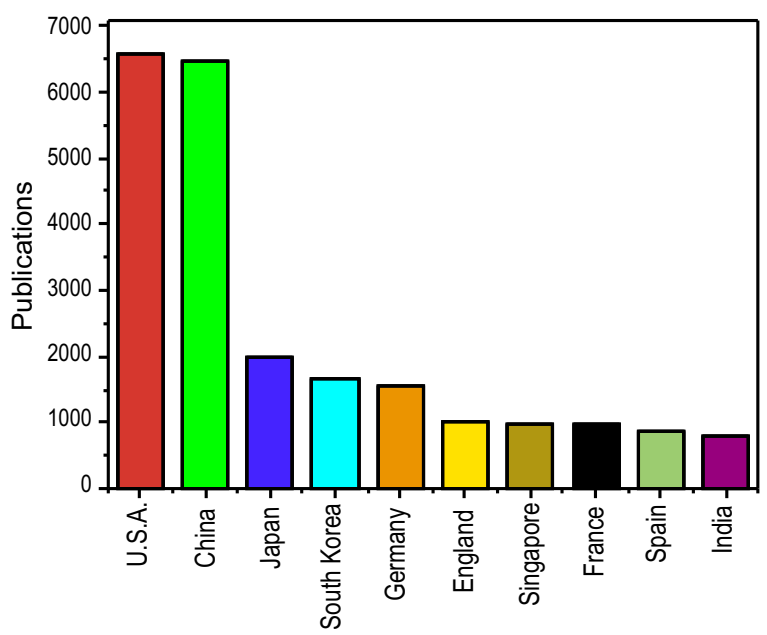

Fig. 3. Country-wise publication development in graphene since 2000-2012 (Dhand et al., 2013). 
Above graphs show the importance of the scope of graphene in field of research, which presents it as an area of keen interest for researchers from two decades. No. of publications for graphene and its oxide from 2008 to 2017 is also reported by Ma et al. (2017) and it shown in Fig. 4.

Synthesis. Graphene can be produced by different techniques as dry exfoliation (which is the piercing of materials in shrilled sheets via forces of mechanical, electromagnetic or electrostatic nature in any environment). Liquid-phase exfoliation (which includes dispersal

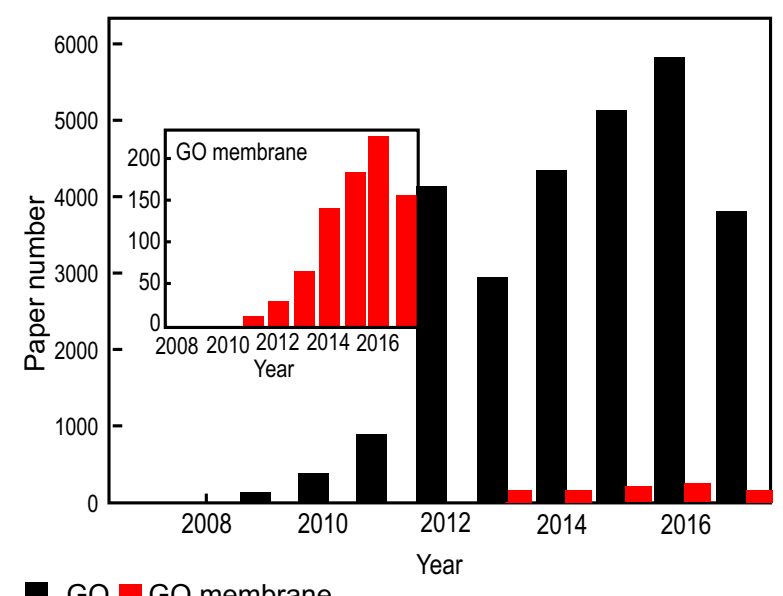

GO GO membrane

Fig. 4. No. of publications for graphene and its oxide from 2008 to 2017 (Ma et al., 2017). of graphite in solvent, followed by exfoliation and then purification), growth on $\mathrm{SiC}$, growth on metals by precipitation, chemical vapour deposition (CVD), thermal CVD on surface of metals, molecular beam epitaxy, chemical synthesis, nano-ribbons and quantum dots. Graphene processing after production goes through the transfer, placement and shaping procedures (Bonaccorso et al., 2012).

Precursors. Several kinds of precursors are used in synthesis of graphene comprising of solid, liquid or gas phases. Gas precursors of hydrocarbons are the common source for carbon due to its advanced purity as associated to other precursors in liquid or solid form. Methane gas is also used as gaseous precursors to develop graphene films (Bera and Maji, 2017). Camphor is used to synthesize graphene although was not a fruitful way but provided pathway to manufacture graphene films by means of solid carbon-based precursors. Hexane is used as source to produce graphene sheets as a liquid carbon precursor. (Randviir et al., 2014).

Catalysts. Transition metals are utilized to produce great quality nanotubes of carbon and graphene. There are many metal catalysts comprising Platinum $(\mathrm{Pt}) 23$, Cobalt (Co) 24, Nickel (Ni) 25-27, Copper (Cu) 28 and others which are used in synthesis of graphene and nanoparticles (Taghioskoui, 2009).

The mechanism of graphene oxide synthesis from the powdered graphene is given in Fig. 5 below illustrating the mechanisms step by step.

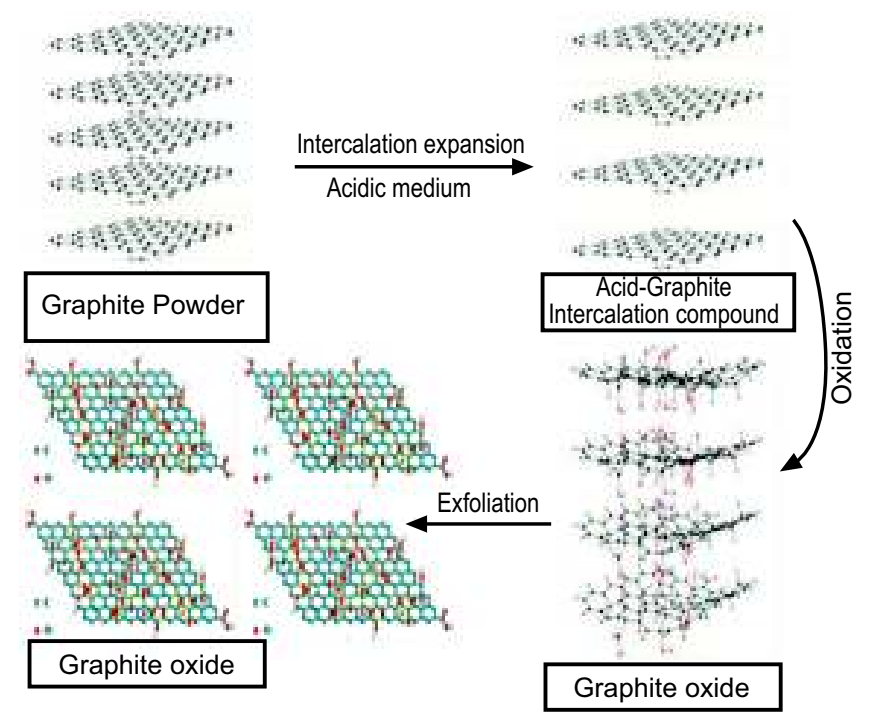

Fig. 5. Synthetic method of GO: oxidation and exfoliation (Ma et al., 2017). 
Characterization. The graphene nanosheets are exposed to many purification phases as water washing, centrifugation, oxidation, drying and acid treatment. Different analyzing techniques are used for analysis of purified graphene sheets such as Raman spectrophotometry, $\mathrm{X}$-ray diffraction, field emission-scanning electron microscopy (FE-SEM) and tunneling electron microscopy. Raman spectrophotometer is used for analysis of nature of chemical bond, any disorder if present, diameter, and flaws in graphene nano sheets. Surface morphology is examined using the field emissionscanning electron microscopy. Tunneling electron microscopy gives the idea about precise size and shape of graphene nano sheets (Lee et al., 2016).

Properties. Graphene holds remarkably great mechanical and electrical characteristics. It displays exceptional optical transparency to an extensive series of light wavelength. Several optoelectronic strategies use as translucent electrodes. In addition, it exhibits splendid flexibility, higher mechanical strength and enormous environmental constancy (Bajenescu, 2012). The honeycomb like graphene lattice comprises of two equal carbon based sub lattices bonded with r-bonds. Every $\mathrm{C}$ atom of lattice with $\mathrm{p}$ orbital contributes in delocalized system of electrons (Penkov et al., 2014).

Nanocomposites of graphene at quite small loading display considerable improvements in its multi-functional features, related to predictable compounds and their derived materials. (Lu et al., 1999) By this, the material not only becomes lighter with easy handling, but also becomes stronger for several multifunctional uses. The amazing properties of graphene can increase its physicochemical abilities of mass matrix on dispersal. It is actually the bonding which commands the rising characteristics of graphene in strengthened nanocomposites (Dhand et al., 2013).

Although electronic characteristics have engrossed lion's share of concern in graphene, the vibrational characteristics have huge significance too. They are accountable for numerous captivating properties such as the thermal conductivity. Graphene comprises of a light atom, wherever inline bonding is much strong, graphene shows high comprehensive velocity. This huge wide-ranging velocity is accountable for great thermal conductivity of graphene which is suitable for many applications. Furthermore, vibrational characteristics are involved in understanding of additional graphene features, comprising optical characteristic through phonon-photon scattering and electronic features through electron-phonon scattering (Cooper et al., 2012).

Get together at the liquid-liquid interface. Atomic self-gathering of carbon nano structures of different measurements is impressive benefits for their potential use in drawing optically straightforward leading terminals, sun based cells, and different gadgets (Wang and Pan, 2010).

Surface property. Graphene has to a great degree of high specific surface territory and extraordinary porosity, to assemble them perfectly for easy adsorption of gases for example, hydrogen $\left(\mathrm{H}_{2}\right)$, methane $\left(\mathrm{CH}_{4}\right)$ and carbon dioxide $\left(\mathrm{CO}_{2}\right)$, (Dhand et al., 2013).

Fluorescence quenching. Graphene have the capacity of fluorescence extinguishing. This feature of graphene can be used for the specific discovery of biomolecules.

Applications. It has wide applications reaching from hydrogen storing devices to batteries. An innovative use of graphene may be ground of electronics. (Si et al., 2018; Zhou et al., 2018). This allows smaller but quicker transistors using fewer energy and scattering heat faster to the silicon grounded devices. Graphene fabricates chemical sensors and transparent layers for solar cells and liquid crystal devices. They show greater sensitivities for detecting single molecules (Lv et al., 2011). The astonishing properties for graphene are investigated for flexible applications going from electronic gadgets towards anode ingredients. It shows exceptional electronic characteristics, allowing power to move quickly through materials. It is demonstrated that electrons of graphene carry massless units like photons, dashing over sheet of graphene without dispersing (Novoselov et al., 2012).

This remarkable electronic property is urgent for some gadget application and graphene could in the long run change silicon ( $\mathrm{Si}$ ) as an element for PC chips, presenting the possibility of ultrafast PCs working at high speeds. (Mukhopadhyay and Gupta, 2011). An enormous potential use for graphene lies in electronic sector. Graphene transistors are estimated to be extremely fast than those made of silicon currently. Silicon has a size boundary wherein the material stops to work (Ramachandran et al., 2013). Nanomaterials connected to graphene have been utilized in Memory devices. Graphene nanomaterials are used frequently. Along with this graphene nanomaterials are also used as transparent electrodes, as light absorbent and electron acceptor, due to their 
good electronic property, ability of transparency and huge specific surface area. Graphene is a right material for applications as metallic transistor. Graphene importers are bipolar by electrons and dumps which can be adjusted by a gateway of electrical field because of distinctive band structure. Sensors monolayer graphene sheet can sense a range of gases and biomolecules (Hansora et al., 2015.

Super capacitors, as transitional power and energy bases among capacitors and batteries, have raised as a vital energy storing technology because of its advanced power supply, high capability and extensive lifespan. They have wide range of applications as power bases in banking hubs, airport controller towers, electric automobiles, clinics, mobile phone towers, logic circuits, and CD players etc. Super capacitors are competent energy storing devices, but have limitations in both price and performance (Basnayaka et al., 2013). It shows exceptional electronic properties, as facilitates the show of electricity speedily from the materials. It has been presented that the electrons of graphene sheets act as massless elements just like photons, burning across the graphene layer preventing its scattering. This exceptional electronic characteristic is essential for several applications (Maidhily et al., 2011).

Graphene it self a nanocomposites and also have excellent prospective in hydrogen storage, that can be utilized in technology of fuel cells, where the fuel cell serve as fuel foundation. Along with these sheets have also being utilized in functionalized sheets as catalyst maintenance for proton interchange membrane fuel cells (Subrahmanyam et al., 2011). Coating for protection is another rising zone where graphene nanocomposites can apply, as graphene has superb chemical confrontation, gas protection, antibacterial property, automated power, lubricity and thermal steadiness. Therefore, it can be improved and varied with different forms of polymers for manufacturing self-cleaning and erosion barrier coverings (Li et al., 2017). Shape memory ability of these polymer is actual decisive for their use in flexible atmospheres, like alteration in temperature, light strength, pressure level, in magnetic and electric fields or the solutions. A variety of polymers can be utilized collectively with various kinds of graphene constituents for diverse shape memory applications (Alhwaige et al., 2013).

IR detectors are another application having great scope for graphene. They can be divided into two distinct types' thermal-based and photon-based detection. In first type, the falling IR radiation is absorbed, resulting in increase of the temperature of the material. This high temperature disturbs some temperature reliant property of the material. In a current study use of the photo thermoelectric effect on graphene to generate electric field is because of electron flow into different metal interactions. Other type is photon-based detectors which use band gap-based recognition with the coming photon which is absorbed and employed to endorse electron hole couples to produce a photocurrent. These detectors can be adjusted to definite wavelengths by generating a quantum sound structure. These IR absorbers are described by having firm absorption rate, but generally want cooling because of thermal effects.

Thermal IR detectors have great response on a large wavelength and can also be used at room temperature but generally have very slow process of absorption mechanism. Therefore, use of graphene based sensors is more appreciable because of great flexibility with minute temperature sensitivity making, it perfect for IR detectors. The capacity to form higher frequency transistors with graphene is promising due to the advanced speed with which the electrons in graphene travel as associated to electrons of silicon. The growth of lithography methods can be utilized to manufacture integrated circuits which based on graphene method.

Thin membranes arranged by means of graphene oxide permits the water to drift over but blocks the dangerous elements and gases. It is thought that it can also be utilized to detoxify sea water at a lesser price than the converse osmosis procedures now in use. A manufacture of operational transistor with graphene is an excellent success meanwhile graphene is not usual semiconductor. While the technical contests, the transistor run at double the speediness of an equivalent silicon transistor. A broadband radio frequency liquidizer is created which is being used in radio requests to develop signals in an array of frequencies. It is a deserted IC element and this success displays that graphene transistors can easily be utilized efficiently in more composite systems.

In indium electrodes graphene is replacing in organic light radiating diodes. The diodes are utilized in electronic scheme to show screens that need little power intake. The material is low-cost and diluter, translucent and analytically is having electric conductance. This makes it perfect for flat-screen demonstrations utilized on smart phones that require electricity to command 
the visual constituents, and to reply to the operator touch. The use of graphene at the place of indium not merely decreases the rate but removes the use of material problems.

Graphene produces an extremely muddy monolayer in vacuum and has a capacity to captivate around $2.3 \%$ of white light. Addition of extra layer of graphene rises the quantity of white light captivated by approx. the same value $(2.3 \%)$. Once photosensitive strength ranges a definite threshold saturable immersion takes place. Because of graphene's characteristics of wavelength independent ultrafast absorption, full group mode securing has been reached. Self-assembly is the property known for an extensive time as the best efficient approaches for nanotechnology. Self-assembly of $2 \mathrm{D}$ graphene sheets is a significant approach for making macroscopic 3D graphene constructions for real uses (Xu et al., 2010).

Actuators. The uncommon optical, mechanical and electrical properties of graphene are being misused by numerous researchers to create actuators. Dou et al. (2013) planned a bilayer paper made out of nearby oxide of graphene and many-sided carbon nanotube layers and showed plainly visible actuators based on graphene.

Supercapacitors or ultra capacitors. Electrochemical capacitors store vitality utilizing moreover particle adsorption or quick surface redox responses or faradaic responses (Domun et al., 2015). They supplement or supplant batteries in electrical energy storing and collecting applications, when extraordinary influenced transport or take-up is essential. An outstanding transformation in execution has been accomplished over later advances in understanding charge stockpiling components and the advancement in cutting edge nanostructured materials (Lin et al., 2008). A battery is high vitality and less power gadget, widely utilized in customary applications, supercapacitor goes about as a less vitality and higher power gadget and is perfect for utilization in higher power beat necessities. An enormous potential use for graphene lies in electronic sector. Graphene transistors are estimated to be extremely fast than those made of silicon currently. Silicon has a size boundary wherein the material stops to work. Its applications are shown in Fig. 6.

Challenges. Although the results of scientific work illustrate excellent benefits of graphene for fast electronics, still the technology is quiet new. It's just

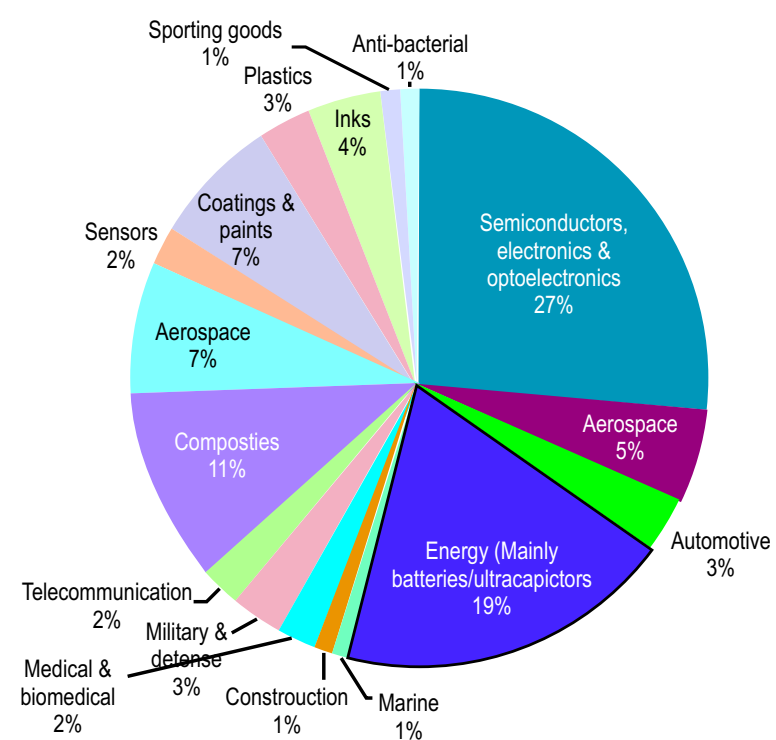

Fig. 6. Wheel showing scope of graphene nano sheets (Somani and Pawar, 2016).

the beginning of technology, and still there is a very long way to go for potential utilization of graphenebased profitable yields. The most important technological test that graphene is facing, the difficulty of precise fabrication of huge sheets. Resolving the problem of mass-production for graphene sheets with good quality is the key attention in the field. Numerous methods have been developed to yield graphene sheets, but the question of strength and reproducibility of these approaches is still questionable. The current infrastructure is consider for the semiconductor industry, the electronics technology is very dependent on silicon. Any approach should be able to adapt itself to the current siliconbased technology. Several patents are being filed on the new techniques and renewed methodologies for its uses. The "certainty" of structural resemblance between carbon nanotubes and graphene is of enormous importance, as carbon nanotubes might be taken as "previous art" in contradiction of graphene in patent uses.

\section{Conclusion}

Although graphene is a novel system of nanomaterial, but still has numerous challenges such as production, applications, characterization, and some others. Due to its controlled modification, oxides of graphene and their reduced form is significant to shield the physical properties to expand their applications. Almost all post 
silicon materials have been ineffectual but graphene is still at phase of improbability, so more detailed study is required to decide if graphene can substitute of silicon. Regardless graphene persists in market or not, but still scientific community is appreciating its discovery. Due to its amazing and unique properties and wide range of applications, it is a favourite area of large number of scientific communities.

Conflict of Interest. The authors declare no conflict of interest.

\section{References}

Alhwaige, A., Agag, T., Ishida, H., Qutubuddin, S. 2013. Biobased chitosan hybrid aerogels with superior adsorption: role of graphene oxide in $\mathrm{CO}_{2}$ capture. Royal Society of Chemistry Advances, 3: 16011-16020.

Bajenescu, T.M. 2012. Trends in graphene research. Electrotehnica, Electronica, Automatica, 60: 7-13.

Basnayaka, P.A., Ram, M.K., Stefanakos, L., Kumar, A. 2013. Graphene/polypyrrole nanocomposite as electrochemical super capacitor electrode: electrochemical impedance studies. Graphene, 2: 81-87.

Bera, M., Maji, P.K. 2017. Graphene-based polymer nanocomposites: materials for future revolution. MOJ Polymer Science, 1: 94-97.

Bonaccorso, F., Lombardo, A., Hasan, T., Sun, Z., Colombo, L., Ferrari, A.C. 2012. Production and processing of graphene and 2D crystals. Materials Today, 15: 564-589.

Booth, T.J., Blake, P., Nair, R.R., Jiang, D., Hill, E.W., Bangert, U., Bleloch, A., Gass, M., Novoselov, K.S., Katsnelson, M.I., Geim, A.K. 2008. Macroscopic graphene membranes and their extraordinary stiffness. Nano Letters, 8: 2442-2446.

Cooper, D.R., D’Anjou, B., Ghattamaneni, N., Harack, B., Hilke, M., Horth, A., Majlis, N., Massicotte, M., Vandsburger, L., Whiteway, E. 2012. Experimental review of graphene. ISRN Condensed Matter Physics, 2012: 1-56.

Dhand, V., Rhee, K.Y., Kim, H.J., Jung, D.H. 2013. A comprehensive review of graphene nanocomposites: research status and trends. Journal of Nanomaterials, 2013: 1-14.

Domun, N., Hadavinia, H., Zhang, T., Sainsbury, T., Liaghat, G.H., Vahid, S. 2015. Improving the fracture toughness and the strength of epoxy using nanomaterials-a review of the current status. Nanoscale, 7: 10294-10329.
Dou, L., You, J., Hong, Z., Xu, Z., Li, G., Street, R.A., Yang, Y. 2013. 25 th anniversary article: a decade of organic/polymeric photovoltaic research. Advanced Materials, 25: 6642-6671.

Gautam, C., Yadav, A.K., Singh, A.K. 2012. A review on infrared spectroscopy of borate glasses with effects of different additives. ISRN Ceramics, 2012: $1-7$.

Geim, A.K. 2009. Graphene: status and prospects. Science, 324: 1530-1534.

Hansora, D.P., Shimpi, N.G., Mishra, S. 2015. Graphite to graphene via graphene oxide: an overview on synthesis, properties, and applications. Journal of Management, 67: 2855-2868.

Hung, W.S., An, Q.F., DeGuzman, M., Lin, H.Y., Huang, S.H., Liu, W.R., Hu, C.C., Lee, K.R., Lai, J.Y. 2014. Pressure assisted self assembly technique for fabricating composite membranes consisting of highly ordered selective laminate layers of amphiphilic graphene oxide. Carbon, 68: 670-677.

Kim, H., Abdala, A.A., Macosko, C.W. 2010. Graphene/ polymer nanocomposites. Macromolecules, 43: 6515-6530.

Lee, H.C., Liu, W.W., Chai, S.P., Mohamed, A.R., Lai, C.W., Khe, C.S., Voon, C.H., Hashim, U., Hidayah, N.M.S. 2016. Synthesis of single-layer graphene: A review of recent development. Procedia Chemistry, 19: 916-921.

Li, H., Song, J., Wang, L., Feng, X., Liu, R., Zeng, W., Huang, Z., Ma, Y., Wang, L. 2017. Flexible allsolid-state super capacitors based on poly aniline orderly nanotubes array. Nanoscale, 9: 193-200.

Lin, Y.M., Jenkins, K.A., Valdes-Garcia, A., Small, J.P., Farmer, D.B., Avouris, P. 2008. Operation of graphene transistors at gigahertz frequencies. Nano Letters, 9: 422-426.

Lu, X., Yu, M., Huang, H., Ruoff, R.S. 1999. Tailoring graphite with the goal of achieving single sheets. Nanotechnology, 10: 269-272.

Lv, P.H., Wang, G.F., Wan, Y., Liu, J., Liu, Q., Ma, F. 2011. Bibliometric trend analysis on global graphene research. Scientometrics, 88: 399-419.

Ma, J., Ping, D., Dong, X. 2017. Recent developments of graphene oxide-based membranes: a review. Membranes, 7: 1-29.

Maidhily, M., Rajalakshmi, N., Dhathathreyan, K.S. 2011. Electrochemical impedance diagnosis of micro porous layer in polymer electrolyte membrane fuel cell electrodes. International Journal of Hydrogen Energy, 36: 12352-12360. 
Mukhopadhyay, P., Gupta, R.K. 2011. Trends and frontiers in graphene-based polymer nanocomposites. Plastics Engineering, 67: 32-42.

Novoselov, K.S., Fal, V.I., Colombo, L., Gellert, P.R., Schwab, M.G., Kim, K. 2012. A roadmap for graphene. Nature, 490: 192-200.

Penkov, O., Kim, H.J., Kim, H.J., Kim, D.E. 2014. Tribiology of graphene: a review. International Journal of Precision Engineering and Manufacturing, 15: 577-585.

Ramachandran, R., Mani, V., Chen, S.M., Saraswathi, R., Lou, B.S. 2013. Recent trends in graphene based electrode materials for energy storage devices and sensors applications. International Journal of Electrochemical Science, 8: 11680-11694.

Randviir, E.P., Brownson, D.A., Banks, C.E. 2014. A decade of graphene research: production, applications and outlook. Materials Today, 17: 426-432.

Rao, C.E.E., Sood, A.E., Subrahmanyam, K.E., Govindaraj, A. 2009. Graphene: the new twodimensional nanomaterial. Angewandte Chemie International Edition, 48: 7752-7777.

Si, Y.H., Xia, Y., Shang, S.K., Xiong, X.B., Zeng, X.R., Zhou, J., Li, Y.Y. 2018. Enhanced visible light driven photocatalytic behaviour of $\mathrm{BiFeO}_{3} /$ reduced graphene oxide composites. Nanomaterials, 8: 1-
12.

Somani, S., Pawar, S. 2016. Graphene: a review. International Research Journal of Engineering and Technology, 3: 375-378.

Subrahmanyam, K.S., Kumar, P., Maitra, U., Govindaraj, A., Hembram, K.P.S.S., Waghmare, U.V., Rao, C.N.R. 2011. Chemical storage of hydrogen in fewlayer graphene. Proceedings of the National Academy of Sciences, 108: 2674-2677.

Sutter, P.W., Flege, J.I., Sutter, E.A. 2008. Epitaxial graphene on ruthenium. Nature Materials, 7: 406411.

Taghioskoui, M. 2009. Trends in graphene research. Materials Today, 12: 34-37.

Wang, L., Pan, Y.T. 2010. Research frontiers and trends in graphene research. New Carbon Materials, 25: 401-408.

Xu, Y., Sheng, K., Li, C., Shi, G. 2010. Self-assembled graphene hydrogel via a one-step hydrothermal process. American Chemical Society Nano, 4: 43244330 .

Zhou, K.G., Vasu, K.S., Cherian, C.T., Neek-Amal, M., Zhang, J.C., Ghorbanfekr-Kalashami, H., Huang, K., Marshall, O.P., Kravets, V.G., Abraham, J. 2018. Electrically controlled water permeation through graphene oxide membranes. Nature, 559: 236240. 